\title{
Something to look forward to
}

\author{
It's tempting to look back, but there's so much more to come.
}

At Nature Physics, we often find ourselves thinking about the past. Anniversaries pop up regularly - 20 years (in 2006) since the discovery of high-temperature superconductivity, 50 years in space (2007), and, earlier this year, the anniversaries of C. P. Snow's 'Two cultures' lecture and of Darwin's On the Origin of Species (the latter celebrated in this journal as an intellectual achievement that still has a bearing on physicists' thinking). In this issue, in two short articles, we're ruminating on the history of quantum mechanics (page 383) and the centenary of a remarkable development in physics: Geiger and Marsden's a-scattering experiment that led to Rutherford's nuclear atom (page 380).

It's fascinating to look back. Physics has a rich history. Following the line of thought through which a concept has been devised and developed - which may span centuries - is often a good route to understanding. It's a history also rich in personality, as many biographies of physicists attest. But it is the past. So, with the twenty-first century touted as 'the century of biology', is it all over for physics?

Hardly. For all those centuries of achievement, there is still so much to do. And the breadth of enquiry is astounding,

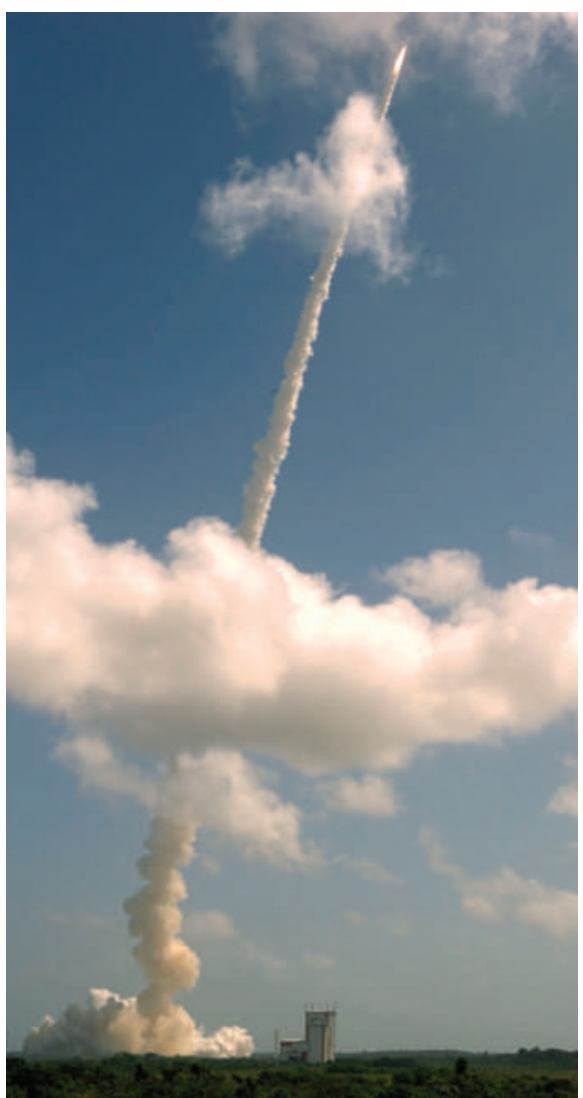

Lift-off: ESA's Planck and Herschel missions begin. from the most intricate of studies on a lab bench, to profound questions about the nature of the Universe - answers to which could soon be beamed back from a satellite more than a million kilometres from Earth. ESA's Planck mission, and Herschel telescope (Planck, Herschel again the backward glance, to honour Max Planck and eighteenth-century astronomer William Herschel), launched faultlessly on board an Ariane 5 rocket from French Guiana last month. Both will orbit the second Lagrangian point, from where Herschel will probe the evolution of stars and galaxies at infrared wavelengths and Planck will map the cosmic microwave background radiation in greater detail than ever before. Science operations begin in the next few months.

It's an over-worn phrase, but both missions are truly a 'new window' on the Universe, and on physics. And that's how it works - finding new windows through which to tackle the questions that have become so well formed through the efforts of physicists of preceding centuries. We seek new windows not only on the grand scale of the Universe, but on the scale of the atom, the electron, the electron spin. And we look forward to finding the answers. After all, every century is a century of physics.

\section{What did you do?}

\section{Nature Physics now requires a statement of authors' contributions to a paper.}

In the past, at the acceptance of a manuscript for publication in Nature Physics, we have gently suggested to the corresponding author that a statement of 'author contributions' be included in the final version. It seemed good practice, and most authors obliged. Now, such a statement is mandatory for any paper published in Nature or a Nature journal.

It's nothing onerous: just a simple listing of the initials of all authors with a comment on what they contributed to the work reported - be it designing, preparing, performing, collecting, analysing, modelling, writing or something else. There is no required format beyond that. These statements can vary greatly in their degree of detail, as is appropriate when the content of Nature Physics papers is so diverse and 'authors' may constitute anything from a lone pair of theorists to a substantial experimental collaboration. (For examples of statements, see http://tinyurl.com/39mmyw.)

It's not hard to imagine, either, why it is a good idea for all papers in the journal to carry an author-contributions statement.
It might serve to ensure that the author list includes only those who really did make a recognizable contribution to the paper. It might be useful for younger scientists, seeking grants or positions, to point to a record of their efforts in producing particular results. It will certainly be beneficial for all concerned, if ever a question of fraud is raised, to know exactly who was responsible for what.

Details of our editorial policies on authorship are available at http://www.nature.com/ authors/editorial_policies/authorship.html. 